\title{
New compact ocean bottom cabled seismometer system deployed in the Japan Sea
}

\author{
Masanao Shinohara • Toshihiko Kanazawa \\ Tomoaki Yamada $\cdot$ Yuya Machida . \\ Takashi Shinbo $\cdot$ Shin'ichi Sakai
}

Received: 18 June 2013/Accepted: 17 September 2013/Published online: 12 November 2013

(C) The Author(s) 2013. This article is published with open access at Springerlink.com

\begin{abstract}
The Japanese islands are positioned near the subduction zones, and large earthquakes have repeatedly occurred in marine areas around Japan. However, the number of permanent earthquake observatories in the oceans is quite limited. It is important for understanding generation of large earthquakes to observe seismic activities on the seafloor just above these seismogenic zones. An ocean bottom cabled seismometer (OBCS) is the best solution because data can be collected in real-time. We have developed a new compact OBCS system. A developed system is controlled by a microprocessor, and signals from accelerometers are 24-bit digitized. Clock is delivered from the global positioning system receiver on a landing station using a simple dedicated line. Data collected at each cabled seismometer (CS) are transmitted using standard Internet Protocol to landing stations. The network configuration of the system adopts two dual methods. We installed the first practical OBCS system in the Japan Sea, where large earthquakes occurred in past. The first OBCS system has a total length of $25 \mathrm{~km}$ and 4 stations with $5 \mathrm{~km}$ interval. Installation was carried out in August 2010. The CSs and single armored optical submarine cable were buried $1 \mathrm{~m}$ below the seafloor to avoid a conflict with fishing activity. The data are stored on a landing station and sent to Earthquake Research Institute, University of Tokyo
\end{abstract}

M. Shinohara (凶) · T. Kanazawa · T. Yamada · Y. Machida · T. Shinbo $\cdot$ S. Sakai

Earthquake Research Institute, University of Tokyo, 1-1-1

Yayoi, Bunkyo-ku, Tokyo 113-0032, Japan

e-mail: mshino@eri.u-tokyo.ac.jp

Present Address:

T. Kanazawa · T. Shinbo

National Research Institute for Earth Science and Disaster

Prevention, 3-1, Tennodai, Tsukuba, Ibaraki 305-0006, Japan by using the Internet. After the installation, data are being collected continuously. According to burial of the CSs, seismic ambient noises are smaller than those observed on seafloor.

Keywords Cabled ocean bottom seismometer . Japan Sea $\cdot$ Earthquake observation $\cdot$ Seafloor observation

\section{Introduction}

The Japanese islands are located near the plate convergent zones, where the Pacific plate and Philippine Sea plate subduct below the Japan islands, and large earthquakes have repeatedly occurred around Japan islands. For studying and understanding the generation processes of these large earthquakes, it is important to observe seismic activities on the sea floor just above these seismogenic zones. A recent pop-up type ocean bottom seismometer (OBS) performs over one-year continuous recording. The long-term OBSs (LTOBSs) are mainly used for an array monitoring of seismic activities in the plate convergent region around Japan (Kanazawa et al. 2009). However popup type OBS has disadvantages such as limited power, data recovery reliability, off-line observation for long-term seismic observation on the seafloor. Although it is an offline observation network, a large scale observation array using a number of LTOBSs is a strong tool for studying earthquakes.

Ocean bottom cabled seismometers (OBCSs), where the sensors are equipped in a hermetically-sealed pressure housing and these cases are connected with cables, has many advantages for seafloor seismic observation. Therefore, OBCSs had been developed based on a submarine 
telecommunication cable system, and have been used over the past 25 years in Japan (e.g. Kanazawa and Hasegawa 1997). However, the OBCS system in the first generation is in-line system and has a small number of seismometers and pressure gauges. For example, an OBCS system installed off Sanriku, Japan, has three seismometers and two pressure gauges.

In 2011, Dense Oceanfloor Network system for Earthquakes and Tsunamis (DONET) (Kaneda et al. 2010) has been installed and started the seafloor observations in the boundary between the source regions of Nankai and Tonanakai earthquakes. The DONET has a main optical fiber cable loop with high reliability and junction boxes connected to the main cable. Various sensors, for example seismometers, pressure gauges, can be connected via the junction box with an underwater mateable connector (UMC). Utilization of the UMCs for scientific sensors enables various type observations and exchanges of the sensors when the sensors have malfunction or upgrade. In addition, a number of the scientific sensors can be increased. The DONET has 20 observation stations. The Neptune Canada regional cabled ocean observatory also has a function of expansion of scientific observations and exchange of sensors (Barnes et al. 2008). However it is difficult to deploy the system quickly, because this type cabled system needs remotely operated vehicle (ROV) for installation or exchange of sensors. Because of complexity of the system, construction and running cost can not be reduced effectively. Since an ROV must manipulate an UMC on a junction box, it is also difficult to bury the whole system below seafloor. This is a problem for avoiding confliction with fishing activity near a coast.

Two cabled ocean-bottom tsunami gauges of the Sanriku OBCS system successfully recorded the tsunami waveform just above the source rupture area of the 2011 off the Pacific coast of Tohoku Earthquake. The tsunami data were essential for estimated the source region of the destructive tsunami by the mainshock (e.g. Fujii et al. 2011; Maeda et al. 2011). Although the existing OBCSs have realized a significant contribution to the study of seismic activity, the number of the equipped seismometers is insufficient for high resolution observations of seismic activities in marine area. After occurrence of the 2011 Tohoku earthquake, it becomes more important to monitor seismic activity and tsunami on the seafloor near source region.

A large problem of the existing OBCS system is construction and running cost. To equip an OBCS system with a sufficient number of seismometers, this problem should be resolved. This is the critical problem in the existing OBCSs. A total cost including production, deployment and maintenance per one observation node for the new system should be less than one third of that for the existing system.
In addition to the problem of construction cost, the existing OBCS has become insufficient for multidisciplinary observation and flexibility of measurements after installation. A portable type system is also required, and is expected to be used for the precise monitoring of seismic activity after large earthquakes. To satisfy these requirements, we adopted the system whose observation nodes are directly connected to seafloor optical fiber cable, i.e. the inline system.

After substantial consideration of interdisciplinary research studies with engineers of various fields, such as ocean engineering, measurement engineering, electronic engineering, mechanical engineering, and information and communication engineering in particular, it was concluded that a new OBCS system using information and communication technologies (ICT) should be developed to resolve the addressed problems, i.e., Internet Protocol (IP) goes to seafloor. According to this concept, we have developed a new OBCS system. The new OBCS system can be assembled compact since a software processes various measurements, while complex and a large amount of hardware are used in the existing OBCSs. Reliability of the system is kept by using redundant system which is easily constructed using the ICT.

There is a tectonic zone where large earthquakes recently occurred in the central coast of the Japan Sea. The new OBCS system was first installed above the source region of the 1964 Niigata earthquake in the Japan Sea in August 2010. Although the deployed OBCS system has a cable length of $25 \mathrm{~km}$ and four cabled seismometers (CSs) with $5 \mathrm{~km}$ spacing, it has been proven that seismic data can be successfully obtained. In this paper, we describe the characteristics, layout, and system parameters of the developed OBCS system, and the characteristics of the seismic data retrieved from the system, especially the ambient seismic noise levels, and performance of the first installed system.

\section{Concept of new OBCS system}

The number of earthquake observatories in the oceans is quite limited on the Earth. Our objective of development is to make observations with a high density array in the marine areas that will be sufficient to achieve the same level of observation as the land-based networks. Because the characteristics of concept of the new OBCS have already been described in detail (Kanazawa and Shinohara 2009; Yamazaki et al. 2012), we summarize its concept here. In original concept, CSs are equipped with optical cables and placed at $20 \mathrm{~km}$ spacing. Optical cables are laid for $900 \mathrm{~km}$ in maximum with a continuous " $\mathrm{S}$ " pattern, by which the $40 \mathrm{CSs}$ are distributed two-dimensionally. The area covered with the conceptual system is comparable 
with a source region of an earthquake with a magnitude of 8. Because the operational depth is planned to $6,000 \mathrm{~m}$, the system can be available for more than $90 \%$ area of the seafloor. The accuracy of the time stamp must be less than $0.1 \mathrm{~ms}$, which is equivalent to the present accuracy of the land seismic network. The system is expected to have an operational lifetime of more than 20 years. This lifetime corresponds to that of the existing system. The most important objective of developing a new OBCS is lowcosts of both production and installation. The size of the CS is a key to achieve this requirement. A smaller CS leads to lower costs for installation. The installation costs by small ordinary ship can be significantly reduced. In addition, the flexibility of measurement is also important for recent multidisciplinary researches. For example, measurement parameters after installation should be changed from the land.

Characteristic of the conceptual network for the OBCS and the land is a doubled ring (Fig. 3 in Yamazaki et al. 2012). This configuration is employed to enable both high reliability and low cost. Ethernet is used as data transmission system for this doubled ring configuration. Data collected with a time stamp at each CS are transmitted using standard IP to landing stations. The landing stations at both end of a cable are equipped with a power supply, a storage system, and access to the Internet. A global positioning system (GPS) clock at each landing station is used as a time reference to synchronize timing of each CS, and is fed to each CS through a dedicated line. Methods of clock transmission have been studied, and it was found that the latest clock synchronization system over Ethernet is sufficient for OBCS. However it was decided that a dedicated optical fiber in addition to optical fibers for Ethernet should be used for clock distribution, because a simple configuration causes high reliability. This dedicated line is also used for control the system of the CS. Under normal operation, the channel for data transmission from each CS to the landing station is chosen to reduce network traffic. When one landing station is not operated for maintenance, the data from all CS can be sent to the other landing station. This operation can be set via IP/Ethernet access from the control station or a landing station. Once an optical fiber submarine cable is broken, data from the CSs can be sent to an individual landing station through an accessible path. The OBCS can be maintained by performance monitoring. The software continuously monitors the status of the system such as the temperature within a pressure vessel, electrical voltages.

Although the existing OBCS consists of hardware only, we decided that a new CS uses a microprocessor for implementation of ICT technologies. This means the new CS is controlled by software which is also capable of processing various measurements. In addition, utilization of software makes the CS compact. Because a central processing unit (CPU) and large scale integration (LSI) can decrease the number of circuits and parts, the cost of the new OBCS can be reduced. In addition, observation parameters can be changed with high reliability from the land. The system and its parts are continuously monitored by software, and we know alert before malfunction such as power down of laser transmitters. We selected Linux operating system (OS) for the OBCS. Although the Linux OS is thought to have enough reliability, the Linux OS can be restarted from landing stations through the dedicated clock line. Additionally, the Linux OS can be accessed from both landing stations using ring configuration of the network.

\section{Development of cabled seismometer for the new OBCS}

The CS for the new OBCS was developed according to the concept we explain before. The Linux OS is able to access two Ethernet lines (Fig. 1). The clock module derives a precise clock generated by a GPS clock from the landing stations, as well as control signals such as Linux restart. The developed Linux board has SH-4 microprocessor which is a widely used in industry. Linux version 2.6.9 is installed as a kernel system. The field-programmable gate array (FPGA), which is a large-scale integrated circuit designed to be configured by using a hardware description language, on the board handles the interface to a digitizer, and stores $30 \mathrm{~ms}$ of data. Linux retrieves data every $30 \mathrm{~ms}$ and then sends it to the landing stations. Two Ethernet switch are also implemented on the FPGA.

The seismometer is a conventional force balance accelerometer (JA-5TypeIII, Japan Aviation Electronics Industry, Ltd.), which is a single axial type. The JA-5 accelerometer has been used for the ocean bottom cable systems developed before in Japan. The accelerometers are orthogonally fixed to the frame in the canister. Because one accelerometer is installed parallel to a seafloor cable, azimuth of this sensor is known during the deployment. The directions of other sensors have to be determined after deployment from a measurement of gravity and particle motions of seismic waves from control sources such as airguns (Machida et al. submitted). Each output of three accelerometers ( $\mathrm{X}, \mathrm{Y}, \mathrm{Z}$ components) is synchronously digitized by 24 bit sigma-delta A/D converters with a sampling rate of $1 \mathrm{kHz}$. Since time is directly stamped to the data on the digitizer using a GPS clock signal from the landing stations, accurate $0.1 \mathrm{~ms}$ time stamping is achieved. The size of the CS is a critical for minimization of the costs. First, we developed electronics unit of the CS as small as possible. There are only 5 boards; CPU, Ethernet switch and clock module (Fig. 2), and two 
Fig. 1 System block diagram of the cable seismometer for the new ocean bottom cable seismometer system. A cabled seismometer has four Ethernet ports for redundancy of communication. SFP small form-factor pluggable, $A D C$ analog to digital conversion, PPS pulse per second, $S W$ switch, $O / E$ optical receiver module, $E / O$ optical transmitter module

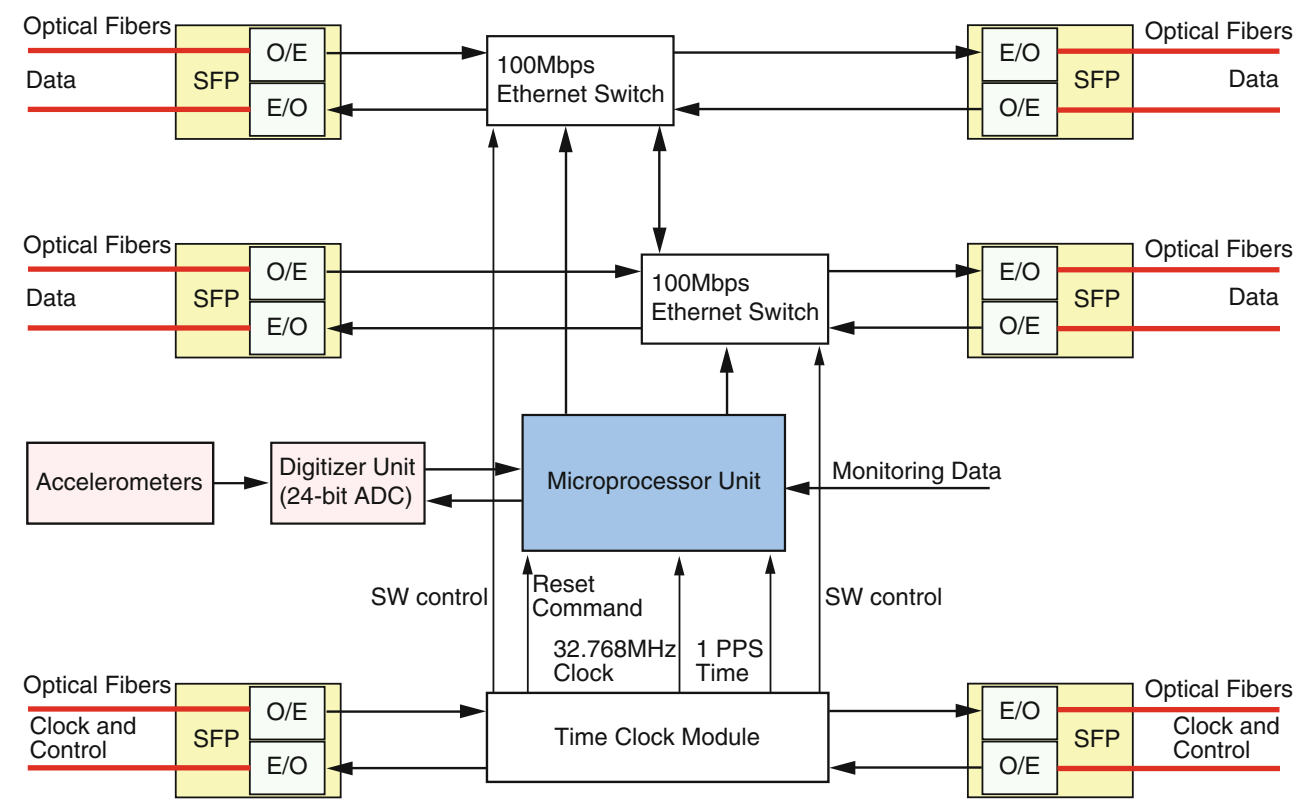

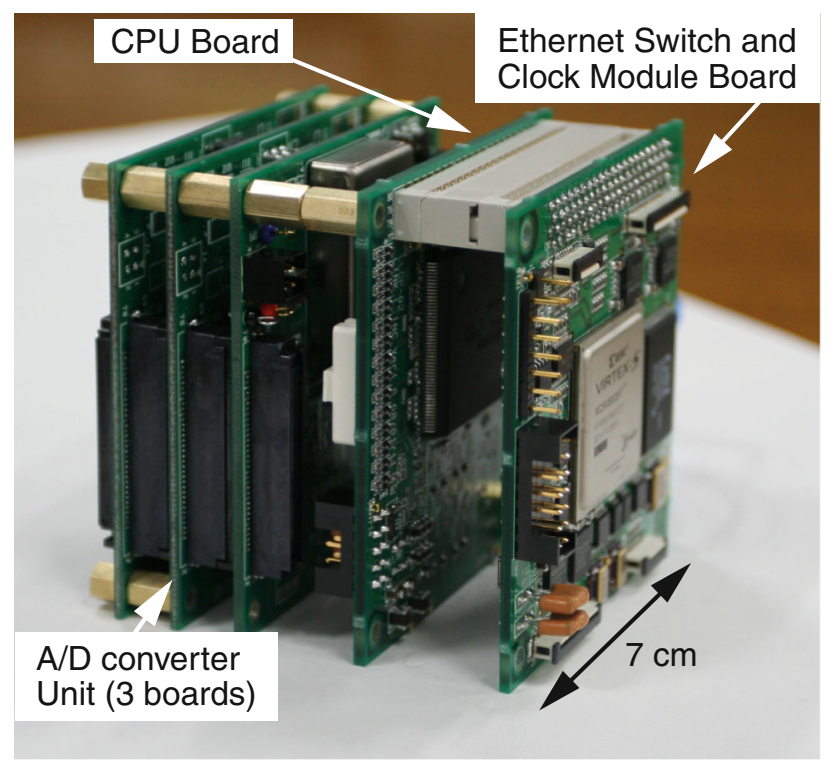

Fig. 2 A photograph of developed central processing unit (CPU) board, Ethernet switch board and analog to digital (A/D) converter boards. Ethernet switch and interface between CPU and A/D are configured by using field-programmable gate array

digitizers in three boards for the CS. Size of each board is approximately $7 \mathrm{~cm} \times 7 \mathrm{~cm}$. In addition to the electronics unit, three seismometers, power unit including zener diodes, and six modules for transformation between optical signals and electrical signals (O/E modules) must be mounted into one package. We adopted small form-factor pluggables (SFPs), which is a compact O/E module used for both telecommunication and data communications. Power for the system is fed from zener diodes through the surge protection circuit. Four zener diodes in serial convert

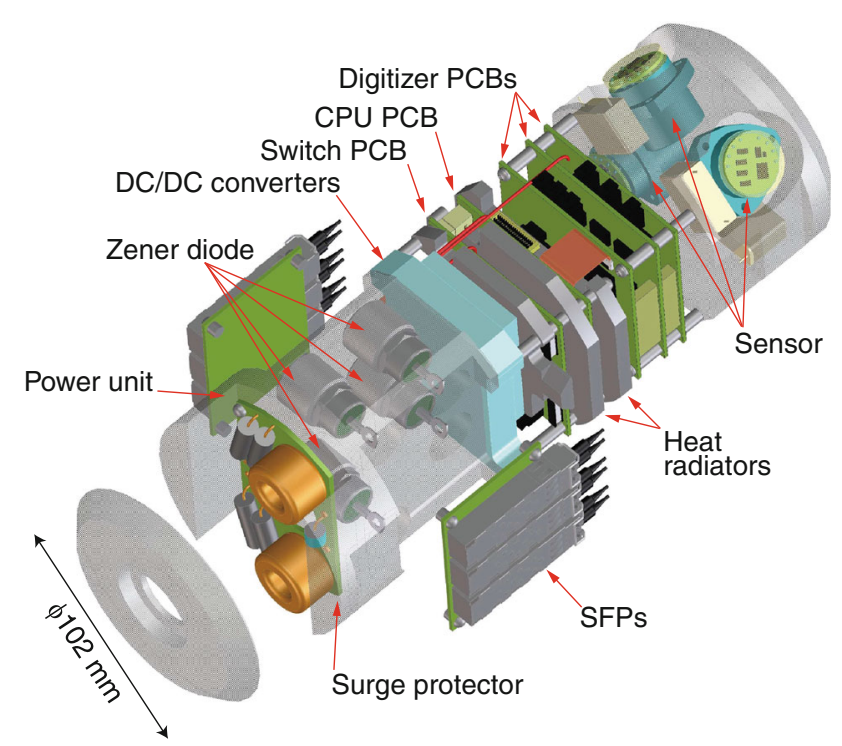

Fig. 3 Rendering of an internal unit of the cabled seismometer. The cabled seismometer package contains three accelerometers, electronics boards, a power unit and small form-factor pluggables (SFPs) for communication. $P C B$ printed circuit board

into electric power with constant voltage (approximately $27.2 \mathrm{~V}$ ). This method using zener diode is reliable and has been used for many commercial submarine cable systems for telecommunication. Various voltages for electronics are generated by using DC/DC converts. Total power consumption of the developed CS becomes approximately $17 \mathrm{~W}$. Figure 3 shows the package of the CS for the new OBCS. A size of the CS package is $10 \mathrm{~cm}$ diameter and $30 \mathrm{~cm}$ long, which is almost equal to that of a $2 \mathrm{~L}$ plastic drink bottle (Fig. 4). A pressure vessel for the new developed CS package was also developed. A Size of the 


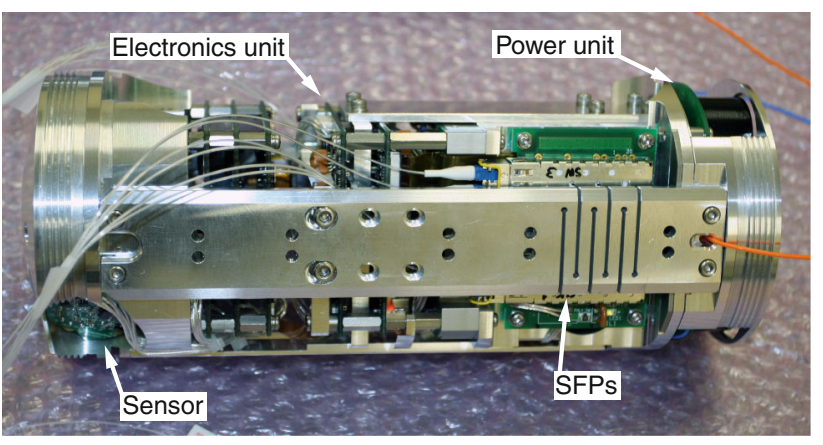

Fig. 4 A photograph of the internal unit of developed cabled seismometer for the new ocean bottom cable seismometer. Metallic frame is directly contact with a part of pressure vessel to conduct heat generated by the electronics

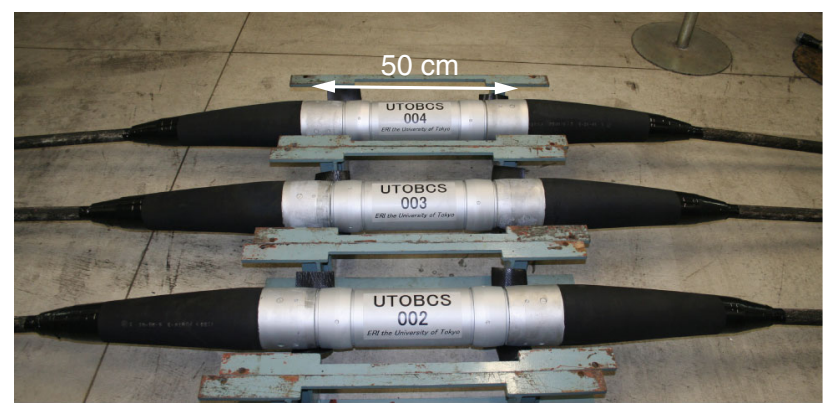

Fig. 5 A photograph of the cabled seismometers for deployment in the Japan Sea. The cabled seismometers have already connected to single armored optical fiber submarine cables. The size of the pressure vessels is $13 \mathrm{~cm}$ in diameter and $50 \mathrm{~cm}$ in length

pressure vessel is $13 \mathrm{~cm}$ in diameter and $50 \mathrm{~cm}$ long (Fig. 5). Due to its small size of the capsule, total size of the CS became smaller than that of the existing CS. Compared to the existing OBCS, which has a $22 \mathrm{~cm}$ diameter and is $150 \mathrm{~cm}$ long, this is a remarkable reduction in size.

\section{Deployment of the first system to the Japan Sea}

Because the development of the CS has been completed, we decide to product a practical system and deploy the new OBCS system in the field. GPS observations with a dense station distribution by the Geographical Survey Institute, Japan revealed that the central coastal area of the Japan Sea has large strain rate, which is named the Niigata-Kobe Tectonic Zone (NKTZ) (Sagiya et al. 2000). Correction is made for the short-term elastic response due to the subduction of the Pacific and Philippine Sea plates (Fig. 6). Historical large earthquakes have occurred in and around the NKTZ, and the large strain rate was estimated to induce the large earthquakes (Sagiya et al. 2000). In the coastal region of the central NKTZ, geological studies indicate that the thick sedimentary basin was formed in a rift structure with a normal fault system that developed during the extension stage of the Japan Sea (Sato 1994). The normal fault system during the rifting stage has been reactivated as a reverse fault system by a change in the tectonic stress from extension to compression. This stress change is estimated to have been caused by variation in the dip angle of the subducting Pacific plate. Recently, the 2004 Chuetsu Earthquake (Sakai et al. 2005), the 2007 Noto-Hanto Earthquake (Yamada et al. 2008), and the 2007 Chuetsuoki Earthquake (Shinohara et al. 2008) occurred in and around the NKTZ. Therefore precise seismic activity which may be induced by the large strain rate should be understood. Although a coastal area in the central NKTZ is close to land where many seismic stations are installed, seismic stations in marine area are needed for studying seismic activities precisely. From these reasons, we decided to install the first practical OBCS system in the source region of the 1964 Niigata earthquake with a magnitude of 7.5, which is the marine area of the NKTZ. Because there is a small island (Awashima) within the source region of the 1964 Niigata earthquake, we decided to construct a landing station for the new OBCS system in Awashima. After a field survey on the Awashima, the system was decided to land at west coast of the island from a view of sustainability of the submarine cable and construction cost.

The new OBCS system for the first installation has a total cable length of $25 \mathrm{~km}$ and $4 \mathrm{CSs}$ with $5 \mathrm{~km}$ spacing. The total length of the system and intervals of the CS are relatively short compared to the conceptual design.Because the target area is small and earthquakes occur at shallow depths, a small size system is effective for earthquake observation in the study area. There is only one landing station due to limitation of the budget. Because the seafloor cable is a bundle of optical fibers, an optical fiber pair for the Ethernet channel is connected to another optical fiber pair at the seaward end of the cable. As a result, the Ethernet channel forms the ring configuration topologically inside single seafloor cable. Since a break of a seafloor cable rarely occurs on the seafloor after deployment, it is considered that a merit of the ring configuration for the data transmission is still kept. Since the optical fiber submarine cable has 8 fibers, three pairs are used for the Ethernet channels and two single fibers are employed for the clock module (Fig. 7). The data transmission channel using the Ethernet is duplicated and one of the Ethernet channels is turned in the furthest CS from the landing station for ring configuration. The clock module also has duplicated channel for redundancy. The data from the CSs are transmitted to the landing station. Under normal operation of the system, the data from 4 CSs can be sent to the landing station with load balancing. When Ethernet switches or CS itself have failure, the data from the CSs can be also sent to 


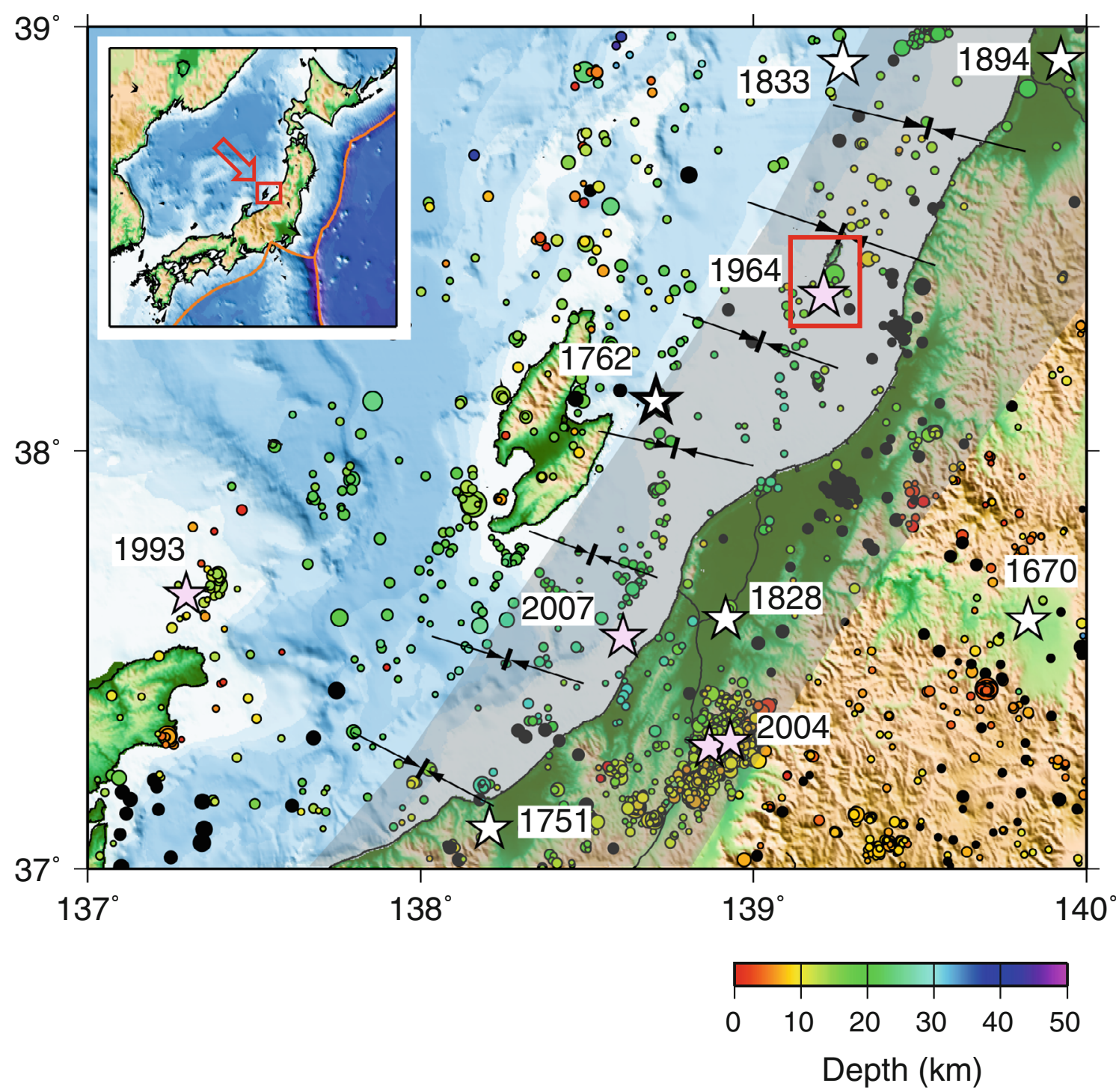

Fig. 6 Epicenter distribution in the Niigata-Kobe Tectonic Zone (NKTZ). Arrows indicate direction and amount of strain (Sagiya et al. 2000). The first OBCS system was deployed off Awashima in the region indicated by the red box and near the 1964 earthquake. Grey shadow region shows the NKTZ estimated from the GPS observation. Stars indicate historical large earthquakes with magnitude greater than 6. Large earthquakes recorded by instruments are indicated as star with light red. Numerals show year of occurring earthquakes.

the landing station through an accessible path. The change of path can be set via IP access or dedicated line for clock from the landing station (Fig. 8). At the landing station, the data are stored in a large disk array system. To transmit the data from the CSs to the data and control center at the Earthquake Research Institute (ERI), University of Tokyo, we decided to utilize Virtual Private Network (VPN). Maintenance of the system is also performed via VPN from a constructor of the system.

Cable route was determined after seafloor surveys which carried out in March and April 2010. At first, outline of the
There are two events labeled as 2004. One is the mainshock and another is the largest aftershock. In 2007, there was the Noto-Hanto Earthquake with a magnitude of 6.9 , however its epicenter is out of this map. Circles denote small earthquakes determined by the Japan Meteorological Agency from July, 2006 to July 2007. Inset is the index map of the study area. Arrow and rectangle show the location of the study area

cable route was determined according to existing data, especially seafloor depth data. The cable was planned to meander for two-dimensional distribution of the CSs. The seafloor surveys were performed by using multi-narrow beam echo sounder, sidescan sonar, subbottom profiler and sampling of sea floor sediments to determine cable route which is suitable for burial of the cable system. Figure 9 shows the cable route for the OBCS with precise seafloor topography as results of the surveys. Generally there is high fishing activity near the shore of the Japan Sea. Because the region of the deployment has water depth less 


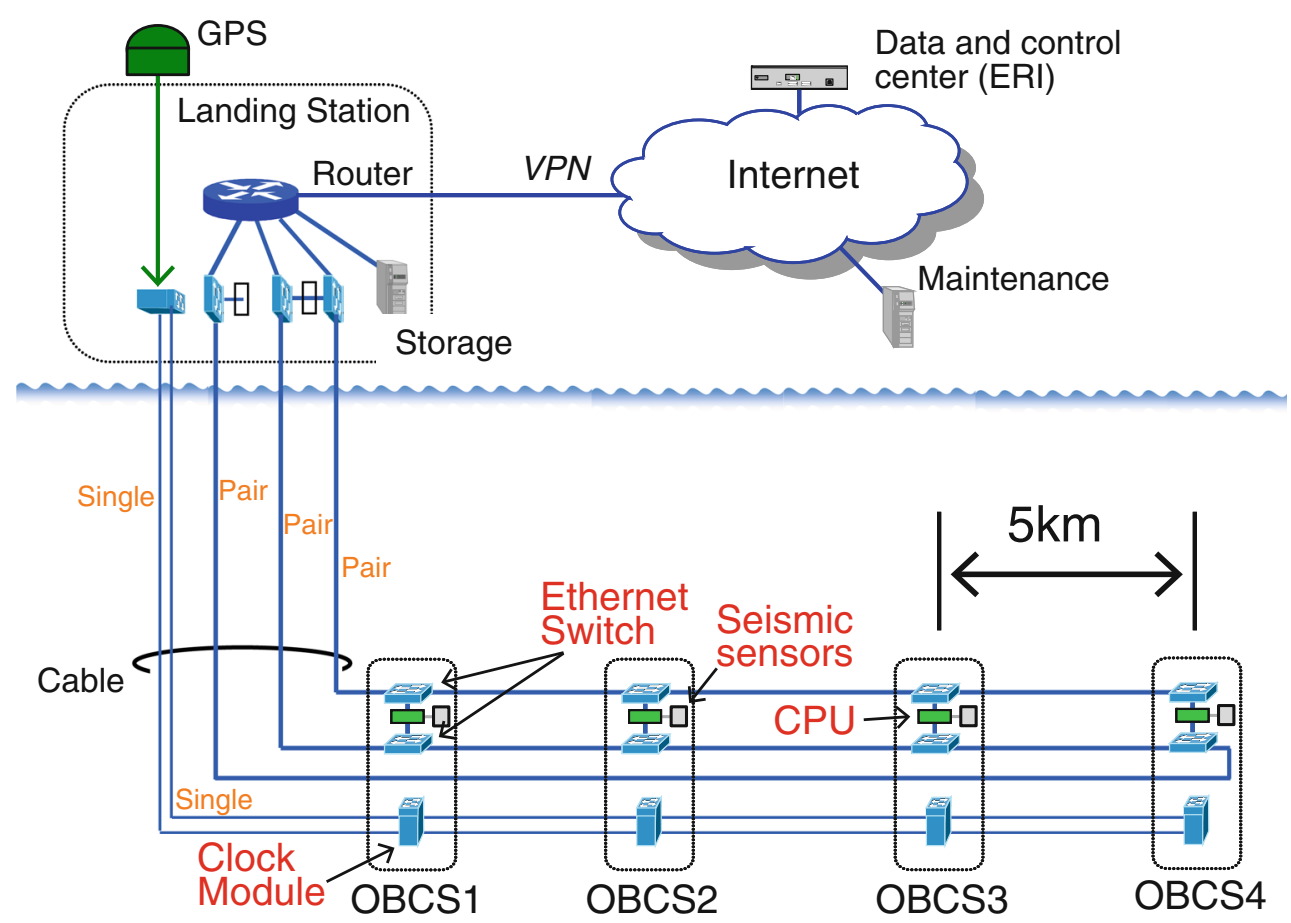

Fig. 7 Network configuration of the OBCS system deployed in the Japan Sea. Transmission redundancy by dual channels and one ring is configured in the system for high reliability. Clock line is also duplicated. Virtual Private Network (VPN) among the landing station, Earthquake Research Institute and a company performing maintenance of the system is established for secure communication
Fig. 8 Three operation modes of the OBCS deployed in the Japan Sea. High reliability is secured by dual-channel configuration. The system also has high maintenancebility

\section{Normal operation}

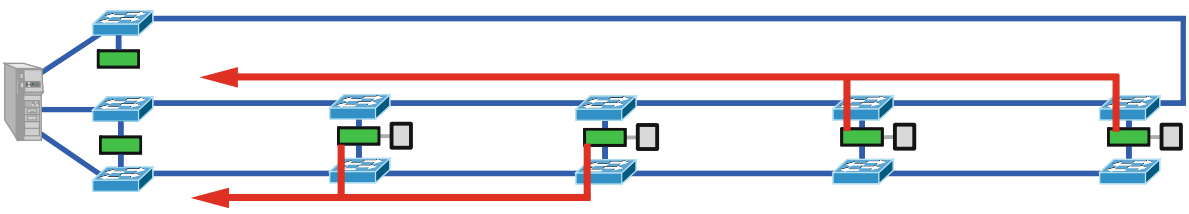

Netwrok switch failure

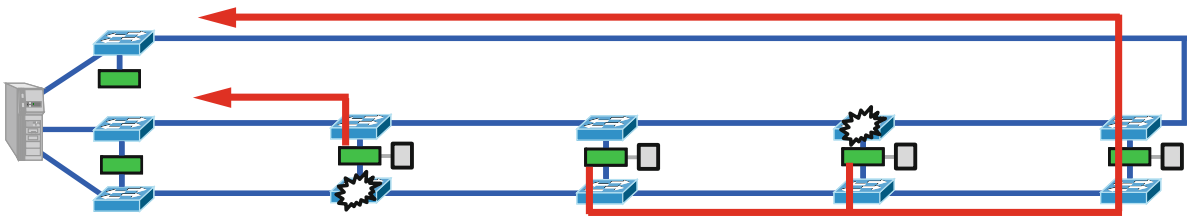

CS failure

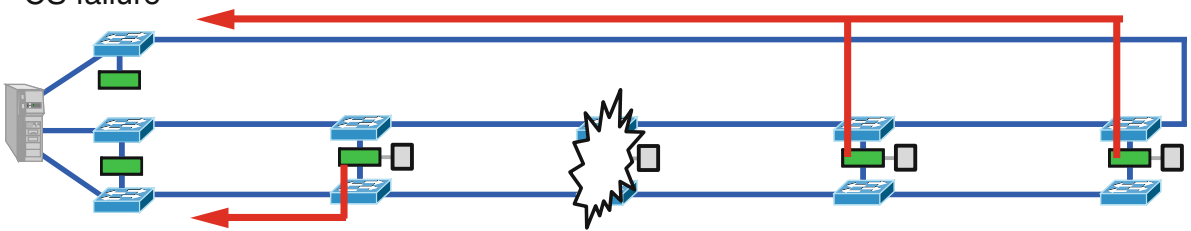

than $110 \mathrm{~m}$, commercial fishing activity is found to be very active. Therefore we decided that we bury the whole system to avoid conflict with fishing activity. Burial of the system protects the submarine cables and the CSs effectively from fishing gear or anchors of large vessels. In addition, there are estimated to be observational advantages for burial of the cabled seismometers. Coupling of the CSs to surrounding crust should be enhanced and a level of ambient seismic noise associated with water current on the seafloor should decrease (e.g. Duennebier et al. 2002; Kaneko et al. 2009). Both contribute to obtain seismic records with a good signal to noise ratio. 


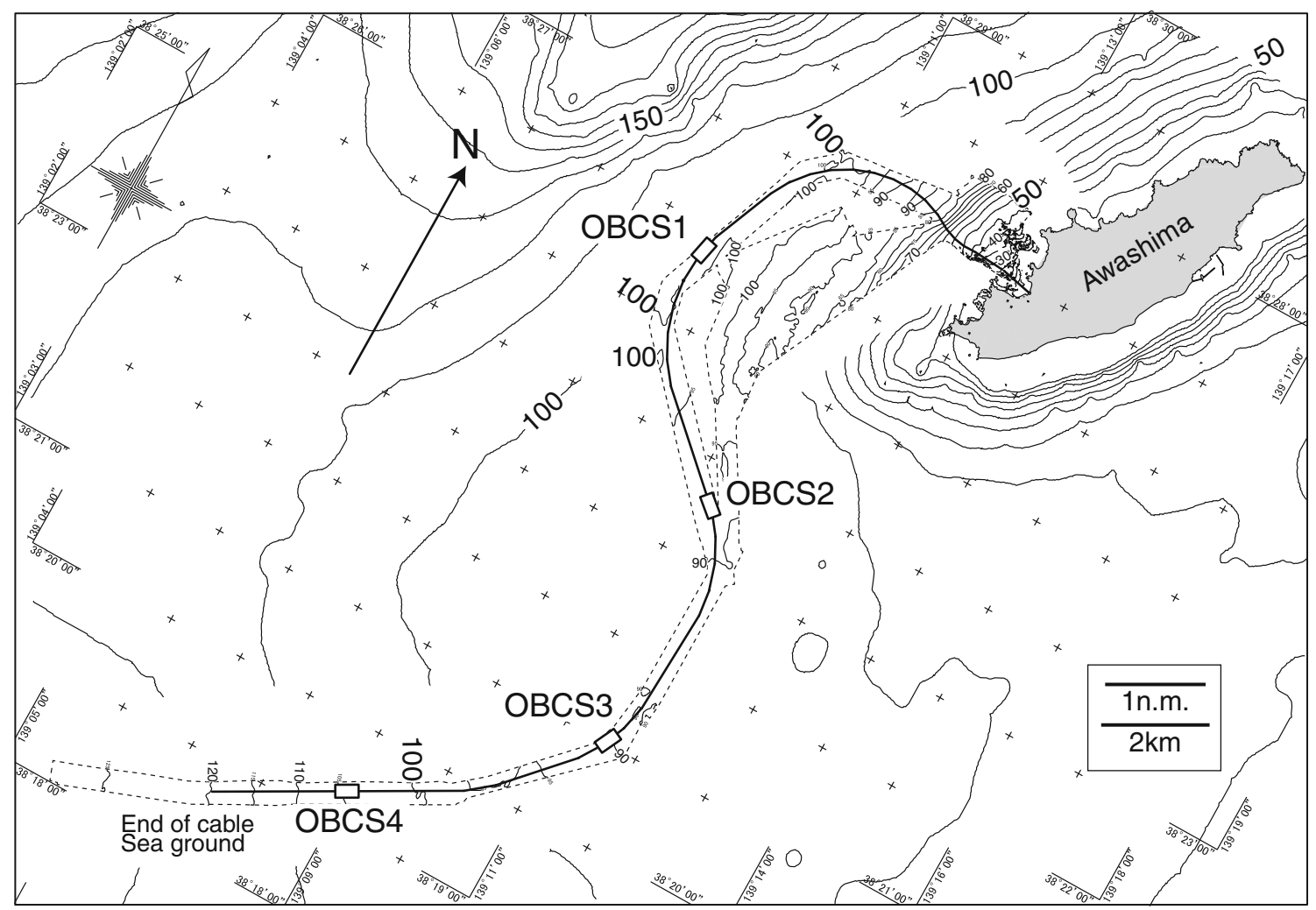

Fig. 9 Position of the cabled seismometers and the cable route with seafloor topography. Numerals indicate water depth. Marine surveys before the deployment were carried out in the region surrounded by dotted line. The system is landed on the west coast of the Awashima, and has a total length of $25 \mathrm{~km}$. Sea ground is installed at the offshore

Deployment of the new OBCS system was carried out from 23th to 28th August, 2010 by using commercial cable ship which is usually used for deployment of submarine cable for telecommunication. On 23th, August, the cable ship swept the seafloor along the cable route to remove obstacles on the seafloor. Cable end was landed to Awashima on 24th, August and the cable ship started deployment of the cable system offshore. The submarine cables and the CSs simultaneously were buried with using a plough-type burial machine. Burial depth is 1 meter below the seafloor (Fig. 10). On 27th and 28th, remote operated vehicle (ROV) buried the submarine cable around the landing point and offshore end of the cable. On 28th August, data recording was started at the landing station. A VPN was configured between the landing station and the Earthquake Research Institute (ERI) University of Tokyo, and data is transmitted to the ERI in real-time and the system is monitored from the ERI.

A landing station was constructed on the west coast of the Awashima, and the submairne cable fed into the landing station. The landing station has GPS receivers, cable termination circuits, computer servers and power supplies for the OBCS system, which are mounted a 19-inch computer rack. Because of a small size of equipment for the landing end of the cable. The landing station is supplying power with minus voltage compared to that of sea water. A part of the system which lies at water depths greater than $30 \mathrm{~m}$ is completely buried $1 \mathrm{~m}$ below the seafloor

station, a building of the landing station has width of $3.2 \mathrm{~m}$, depth of $2.2 \mathrm{~m}$ and a height of $2.7 \mathrm{~m}$ with air-conditioner. This small size of the landing station is also contributed to low cost of the system. The landing station is supplying the power with minus voltage compared to that of sea water at a constant current. Supplying current is approximately $0.75 \mathrm{~A}$ corresponding to a voltage of $130 \mathrm{~V}$.

The Awashima is linked to the mainland Honshu by microwave radio communication at the present. We found that capacity of the Internet connection is not enough to send all the data from the OBCS system. Therefore the system status of the OBCS and a part of the data are sent to the ERI. The data from the seismometers are decimated at a sampling frequency of $100 \mathrm{~Hz}$ for real-time transmission. When a remarkable event occurs, all the data of the event will be able to be retrieved via protocol of the ftp. Commands controlling the system are sent from the ERI.

\section{Seismic records from the OBCS system}

The seismic data from the OBCS enable us to study the seismic noise in the frequency range from $10 \mathrm{mHz}$ to 


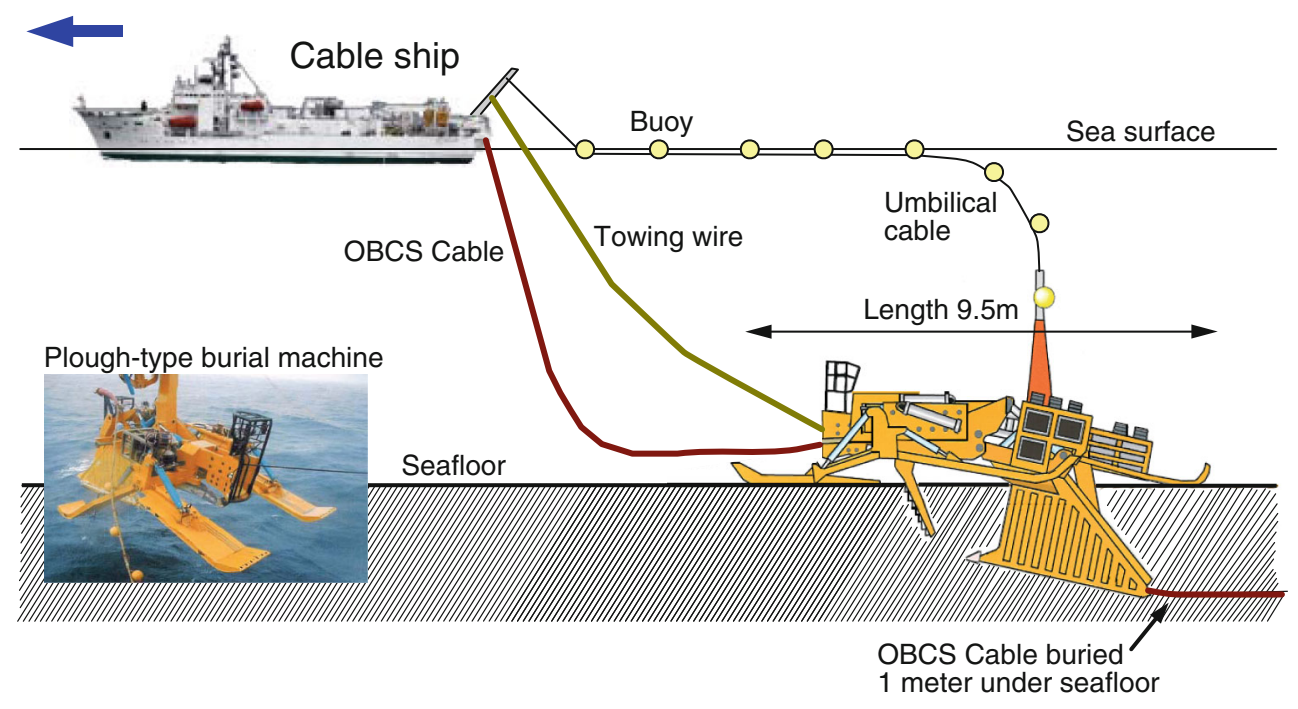

Fig. 10 Simultaneous operation of deployment and burial of the submarine cables and the cabled seismometers using a plough-type burial machine. Laying cables and cabled seismometers under the seafloor protects them effectively from fishing gear or anchors of

$20 \mathrm{~Hz}$ in the shallow water depth. Spectrum of the ambient seismic noise is calculated with a time window of about $250 \mathrm{~s}$ (Fig. 11). It is found that the noise levels at the OBCS reach $-130 \mathrm{db}$ (reference of $1 \mathrm{~m}^{2} / \mathrm{s}^{4} / \mathrm{Hz}$ ) in frequency greater than $3 \mathrm{~Hz}$ and around $0.2 \mathrm{~Hz}$. This level of ambient seismic noise is comparable to a noise floor of the used accelerometer. On the other hand, small changes of noise levels in frequency around $1 \mathrm{~Hz}$ are seen. The ambient seismic noise levels are smaller than High Noise Model (Peterson 1993) at frequency greater than $0.1 \mathrm{~Hz}$. The noise of the OBCS is considered to be much smaller than that recorded on the seafloor at such a shallow water depth. This indicates that the burial seismic sensor below the seafloor is effective to reduce a seismic noise in an environment of shallow water depth. During seismic observation on the seafloor in open sea, a large seismic noise around a few seconds known as microseisms is observed (e.g. Araki et al. 2004; Shinohara et al. 2006). From the records of the OBCS, dominant frequency of large ambient noise is around the frequency of $1 \mathrm{~Hz}$. A size of the Japan Sea which is small compared to the oceans may cause this difference. Speed of wind on the Earth surface is considered to increase seismic noise level at high frequency $(>0.1 \mathrm{~Hz}$ ) (McCreery et al. 1993; Wilcock et al. 1999; Collins et al. 2001). A meteorological observation is being operated on the Awashima by Japan Meteorological Agency (JMA). We compare the noise spectra of the OBCS with average wind speed (Fig. 11). Wind speed seems to be related with the noise level for frequency around $1 \mathrm{~Hz}$.

Waveform data from the OBCS system are continuously recorded to the disk array at the sampling frequency of $1 \mathrm{kHz}$ (Fig. 12). The decimated data to a sampling large vessels. The developed cabled seismometer is small enough to pass through the plough-type burial machine. A part of this figure by courtesy of Kokusai Cable Ship Co., Ltd

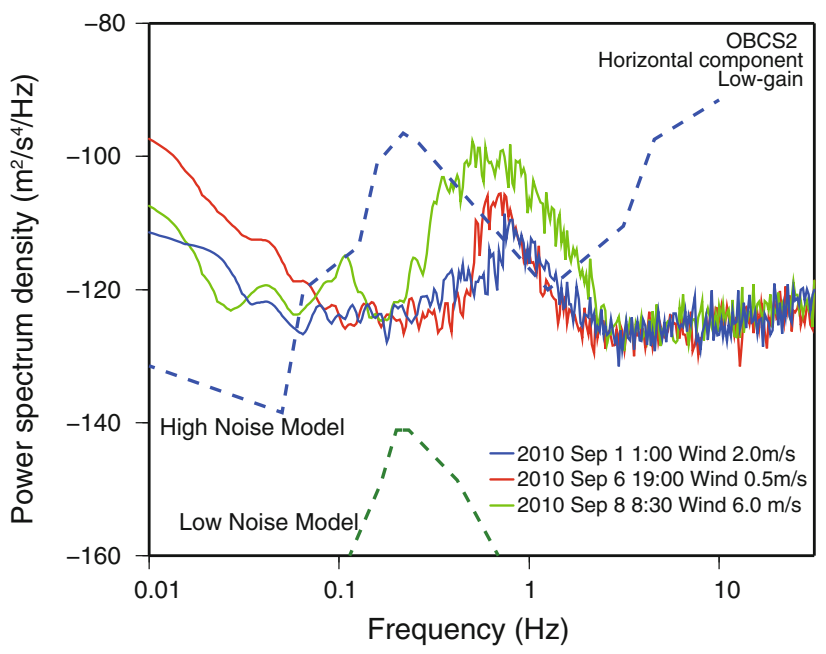

Fig. 11 Power spectra of the OBCS system in the Japan Sea. Power spectra estimated using approximately $250 \mathrm{~s}$ records for OBCS2 are plotted. The high noise model and the low noise model by Peterson (1993) are also shown. Three power spectra which have different meteorological circumstance are estimated for horizontal component of low gain channel. A level of ambient seismic noise around $1 \mathrm{~Hz}$ depends on speed of wind on the surface

frequency of $100 \mathrm{~Hz}$ are also sent to the ERI using VPN in real-time. Therefore we can estimate hypocenter of earthquakes occurring near the Awashima. The JMA had determined event positions using the data of the permanent telemetered land seismic network operated by the National Research Institute for Earth Science and Disaster Prevention (NIED), JMA, and universities (the JMA unified hypocenter catalog). We selected events whose epicenter is located below the OBCS from 29th August, 2010 to 31st 


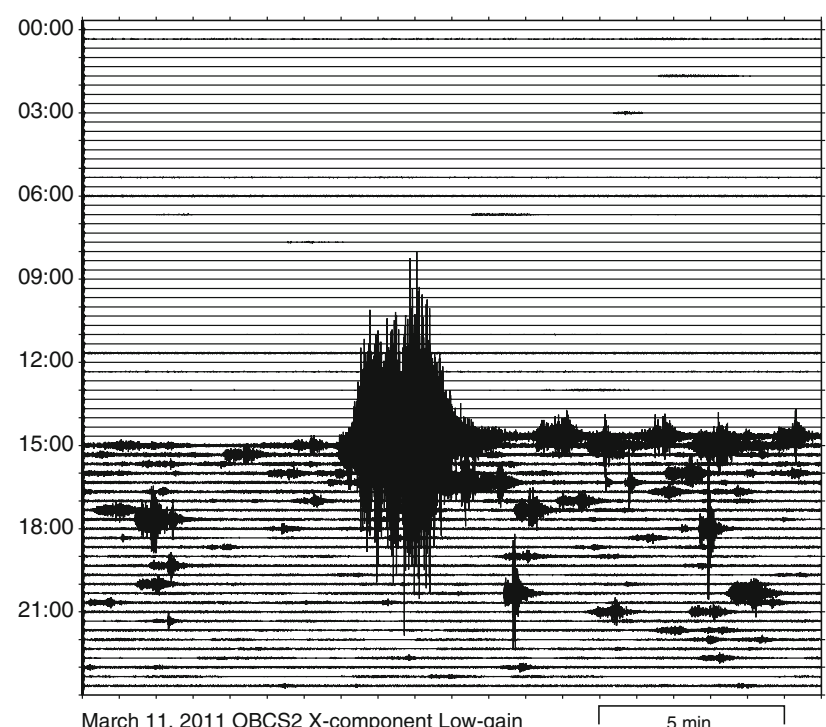

Fig. 12 Seismic records of X-component data of OBCS2 on March 11th, 2011. Each trace is $20 \mathrm{~min}$ long and filtered with a pass band between $1 \mathrm{~Hz}$ and $8 \mathrm{~Hz}$. Time coordinate is Japan Standard Time (UTC +9 h). A large event is the mainshock of the 2011 Tohoku earthquake and many aftershocks followed the mainshock

May, 2012. P and S-wave arrival times were picked on a computer display (Urabe and Tsukada 1991) (Fig. 13). For picking up arrivals, we used 4 CSs and a land seismic station on the Awashima operated by JMA. We selected 45 events with more than three P-wave arrival readings and more than one $\mathrm{S}$-wave reading from the network. Hypocenters were determined by a maximum-likelihood estimation technique of Hirata and Matsu'ura (1987). For the location, we use one-dimensional velocity structure and station correction values estimated by seismic survey carried out in 2012 (Machida et al. submitted). We assumed that the ratio of $\mathrm{P}$-wave to $\mathrm{S}$-wave velocities in all layers is 1.73. The networks located 22 earthquakes with an error of less than $1 \mathrm{~km}$ in the horizontal direction and less than $2 \mathrm{~km}$ in depth (Fig. 14). The estimation errors of each hypocenter location were calculated from the total covarience matrix of the location program (Hirata and Matsu'uura 1987). Because we selected the events from the JMA unified hypocenter catalog, we can compare hypocenters which are located by the OBCS data to those determined from the data of the land seismic network by the JMA (Fig. 14). Differences of epicenters are relatively small, however, depths of events have large differences. The depths of the events by the OBCS data generally become 4-10 km shallower. Because the velocity structure for our location has lower velocity in the region shallower than $3 \mathrm{~km}$ than that used for the JMA location, our location has tendency to have shallower depths of the events. However, the events become shallower than estimation from the difference of the velocity structures. This means

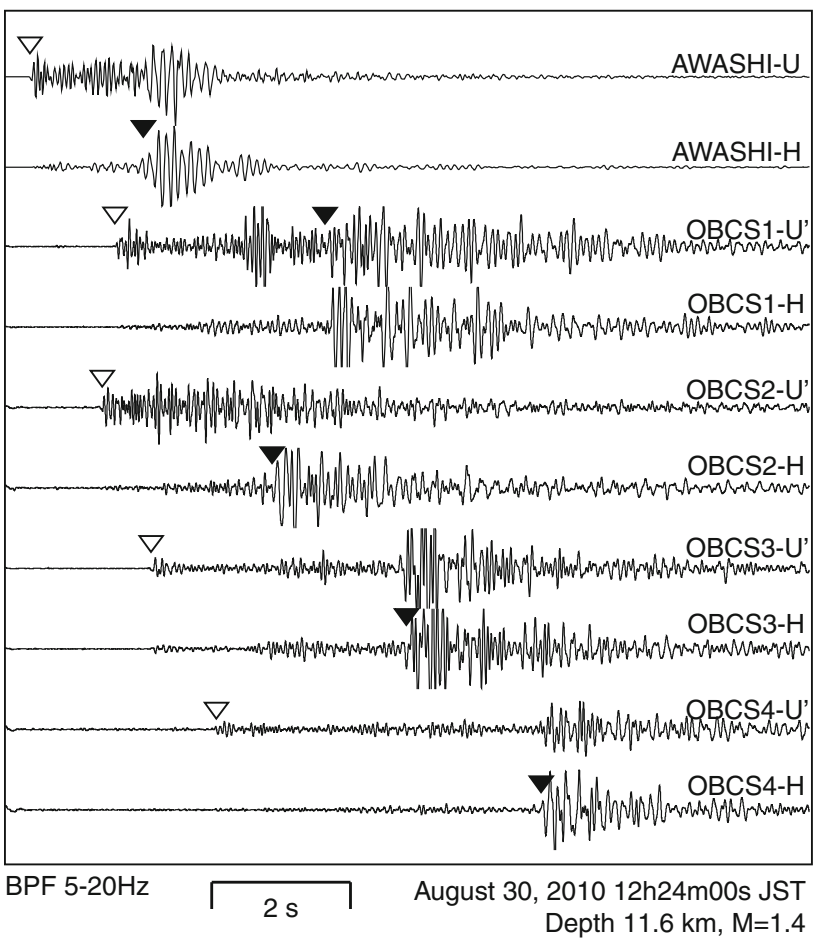

Fig. 13 Example of seismograms of an earthquake for the OBCSs and a land station on the Awashima. See Fig. 14 for positions of the OBCSs and the land station on the Awashima. Two components, which are equivalent to vertical and horizontal components, are shown and band-pass filter with $5-20 \mathrm{~Hz}$ is applied. The origin time, focal depth and magnitude are shown. Epicentral distances to stations range approximately $10-30 \mathrm{~km}$. Solid and open inverted triangles indicate $\mathrm{P}$ - and $\mathrm{S}$-wave arrivals, respectively

that positions of the located earthquakes by the OBCS data is thought to have high resolution.

The study area corresponds to the source region of the 1964 Niigata earthquake with magnitude of 7.5. The located hypocenters seem to form a dipping plane toward the west at an angle of $34^{\circ}$. From the analysis of focal mechanism of the mainshock, an angle of westward dipping nodal plane is $70^{\circ}$ (Aki 1966). In addition, Abe (1975) estimated an angle of the plane is $56^{\circ}$ from focal mechanism and geodetic data. The present seismic activity is not thought to relate directly to the seismic activity of the mainshock in 1964. Further continuous observation is needed to reveal a relation between the present seismic activity and the fault plane of the 1964 mainshock.

\section{Conclusions}

The new compact OBCS with ICT technology was developed. The application of ICT technologies makes it possible to realize remarkable and new features. ICT has enabled the new OBCS to become more compact and less expensive, and enabled IP access and the upgrade of OBCS 

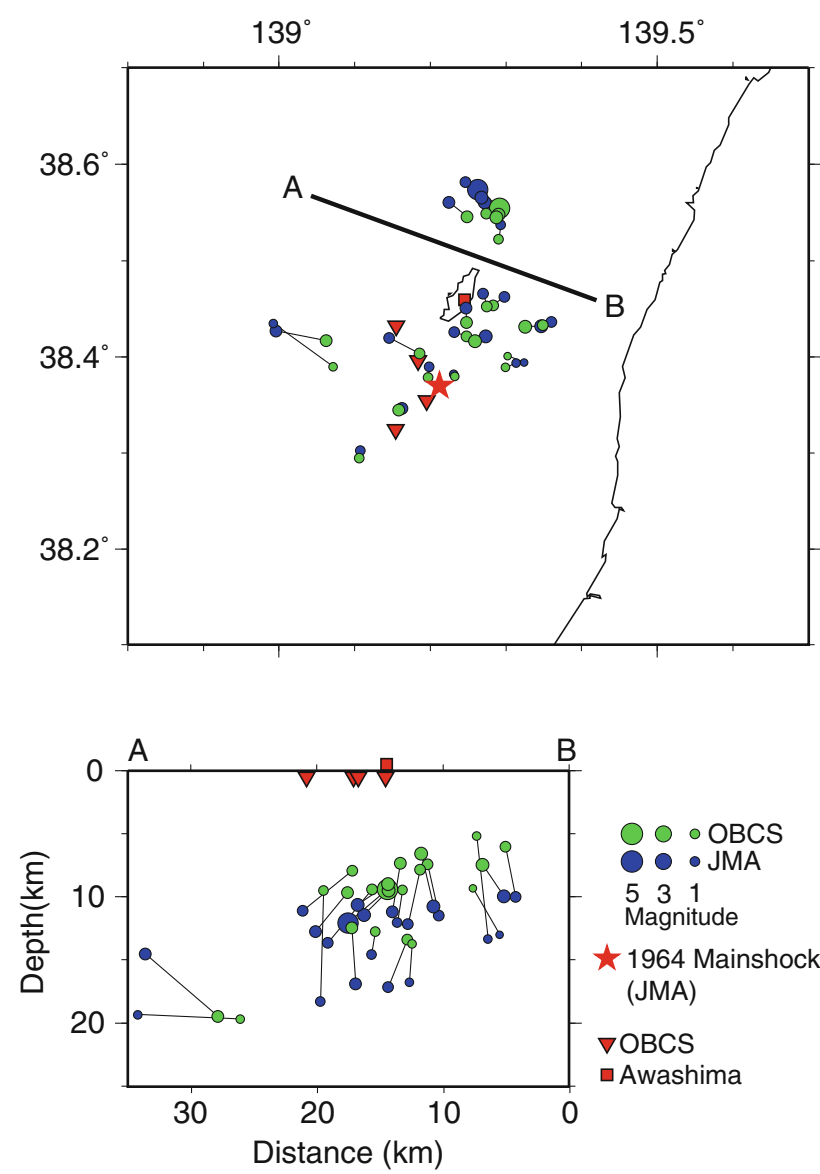

Fig. 14 Hypocenter distribution (August 30th, 2010-May 31st, 2012) derived by using the OBCS. The inverted red triangles and red square indicate the positions of the cabled seismometer and the land seismic station on the Awashima, respectively. The circles filled with green represent hypocenters determined by the OBCS data. The diameters of the circles are proportional to the magnitudes. Blue circles indicate hypocenters determined by the JMA for the same period. To compare hypocenters by the OBCS and those determined by the JMA, corresponding events are connected by line. Vertical hypocenter distribution projected onto profile $a-b$ is also shown

for the flexibility and expandability of measurements. The CS of the new compact OBCS can be made so compact since software processes various measurements, while complex and a large amount of hardware are used in the existing OBCS, and lowered the costs for both production and installation. Reliability of the system is kept by using redundant system which is easily constructed using the ICT. The CSs of the OBCS on the seafloor can be accessed through IP protocol from UNIX systems on land. This will provide us an ability of changing measurement parameters of the seismometers, and upgrading the installed firmware of the FPGAs and software in the CSs.

A microprocessor controls the whole system of the CS and TCP/IP is used for communication. The CS has four Ethernet switches which are implemented on FPGA to change a communication path. The system is controlled by Linux OS. Signals from three accelerometers are digitized with a resolution of 24-bit at a sampling frequency of $1 \mathrm{kHz}$. The clock signal is sent by using GPS from the landing station. A size of the developed CS is $13 \mathrm{~cm}$ in diameter and $50 \mathrm{~cm}$ long.

The central coastal area of the Japan Sea has large strain rate, and had large earthquakes in past. The first system has a total length of $25 \mathrm{~km}$ and 4 CSs with $5 \mathrm{~km}$ interval and was deployed in the coastal area of the central part of the Japan Sea, where a large earthquake occurred in 1964. The cable route was decided by using results of marine surveys. The whole system was buried 1 meter below the seafloor to avoid a conflict with fishing activity. The data are stored on a landing station and sent to the ERI, University of Tokyo by using the Internet in real-time.

After the installation, data are being collected continuously. According to burial of the seismometers, seismic ambient noises are smaller than those observed on the seafloor. The level of ambient seismic noise recorded by the deployed OBCS is comparable to a noise floor of the used accelerometer. Wind on the surface affects the noise level for frequency around $1 \mathrm{~Hz}$. We relocated hypocenter of earthquakes occurring near the Awashima using the OBCS data. The depths of the events become 4-10 km shallower than those determined by a land network, because spatially high density observation gives results with high resolution. The system has collected continuous seismic data for three years since the deployment. We will continue to gather data from a view of both scientific and technological researches.

Acknowledgments The success of the development and first deployment was made possible by the active co-operation of many scientists, engineers, technicians from various institutions. In particular, the authors express thanks to Drs. H. Utada, Y. Morita, H. Shiobara, K. Mochizuki, Messrs. T. Yagi, Y. Hirata, S. Hashimoto from Earthquake Research Institute, University of Tokyo, Dr. K. Yamazaki from Nagaoka University of Technology, Mr. Y. Shirasaki from Marine Eco Tech Ltd., Mr. J. Kojima from KDDI R\&D Labs., Inc., Ms. Y. Jyono, Messrs. K. Yamamoto, H. Kainuma, and S. Chiba from LINK Lab. Inc., Mr. K. Furukawa from Intertechno Co., Ltd., Messrs. R. Morikawa and H. Shirani from OCC Corp., Messrs. N. Fukushima and T. Etoh from KCS Co., Ltd., Dr. K. Asakawa from JAMSTEC, who have all made substantial contributions to the development and deployment of the new OBCS system. We are also grateful to two anonymous reviewers for their critical reviews for improvement of this manuscript. This study is partly supported by the Ministry of Education, Culture, Sports, Science and Technology (MEXT) of Japan, and by the cooperative research program of the Earthquake Research Institute, University of Tokyo. Most of the figures were created using GMT (Wessel and Smith 1991).

Open Access This article is distributed under the terms of the Creative Commons Attribution License which permits any use, distribution, and reproduction in any medium, provided the original author(s) and the source are credited. 


\section{References}

Abe K (1975) Re-examination of the fault model for the Niigata Earthquake of 1964. J Phys Earth 23:349-366

Aki K (1966) Generation and propagation of $G$ waves from the Niigata earthquake of June 16, 1964. Part1. A statistical analysis. Bull Earthq Res Inst Tokyo Univ 44:23-72

Araki E, Shinohara M, Sacks S, Linde A, Kanazawa T, Shiobara H, Mikada M, Suyehiro K (2004) Improvement of seismic observation in the ocean by use of seafloor boreholes. Bull Seismol Soc Am 94:678-690

Barnes CR, Best M M R, Zielinski A (2008) The NEPTUNE Canada regional cabled ocean observatory-An overview of the progress of installation and the design of the science experiments. Sea Technol 49(7):10-14

Collins JA, Vernon FL, Orcutt JA, Stephen RA, Peal KR, Wooding FB, Spiess FN, Hildbrand JA (2001) Broadband seismology in the oceans: lessons from the ocean seismic network pilot experiment. Geophys Res Lett 28(1):49-52

Duennebier FK, Harris DW, Jolly J, Babinec J, Copson D, Stiffel K (2002) The Hawaii-2 Observatory seismic system. IEEE J Oceanic Eng 27(2):212-217

Fujii Y, Satake K, Sakai S, Shinohara M, Kanazawa T (2011) Tsunami source of the 2011 off the Pacific coast of Tohoku Earthquake. Earth Planets Space 63:815-820

Kanazawa T, Hasegawa A (1997) Ocean-bottom observatory for earthquakes and Tsunami off Sanriku, North-Eastern Japan using submarine cable. In: Proceedings of international workshop on scientific use of submarine cables, Okinawa, pp 208-209

Kanazawa T, Shinohara M (2009) A new, compact ocean bottom cabled seismometer system-Development of compact cabled seismometers for seafloor observation and a description of first installation plan. Sea Technology 50(7):37-40

Kanazawa T, Shinohara M, Shiobara H (2009) Recent progress in seafloor earthquake observations and instruments in Japan. Zishin 2(61):S55-S68 (in Japanese with English abstract)

Kaneda Y, Kawaguchi K, Araki E, Matsumoto H, Nakamura T, Kamiya S, Ariyoshi K, Hori T (2010) Dense ocean floor network system for mega thrust earthquakes and tsunamis(DONET) towards understanding mega thrust earthquakes. SubOptic. http://www.suboptic.org/uploads/Files/208_Oral_THU_1B_03. pdf

Kaneko S, Araki E, Kawaguchi K, Sakuma A, Matsumoto H, Kodera $\mathrm{T}$, Kaneda Y (2009) Installation method of high-quality seismic observation in the seafloor. OCEANS'09 IEEE Bremen

Machida Y, Shinbo T, Shinohara M, Yamada T, Mochizuki K, Kanzawa T (2013) A seismic refraction survey conducted for the Ocean Bottom Cable Seismometer system. J Jpn Soc Mar Surv Tech (in Japanese with English abstract) (submitted)
Maeda T, Furumura T, Sakai S, Shinohara M (2011) Significant tsunami observed at the ocean-bottom pressure gauges at 2011 Off the Pacific Coast of Tohoku Earthquake. Earth Planets Space 63:803-808

McCreery CS, Duennebier FK, Sutton GH (1993) Correlation of deep ocean noise $(0.4-30 \mathrm{~Hz})$ with wind, and the Holu Spectrum-A worldwide constant. J Acoust Soc Am 93:2639-2648

Peterson J (1993) Observations and modeling of seismic background noise. Open-File Report 93-322, US Department of Interior Geological Survey

Sagiya T, Miyazaki S, Tada T (2000) Continuous GPS array and present-day crustal deformation of Japan. Pure Appl Geophys 157:2303-2322

Sakai S, Hirata N, Kato A, Kurashimo E, Iwasaki T, Kanazawa T (2005) Multi-fault system of the 2004 mid-Niigata prefecture earthquake and its aftershocks. Earth Planets Space 57:417-422

Sato H (1994) The relationship between late Cenozoic tectonic events and stress field and basin development in northeast Japan. J Geophys Res 99:22261-22274

Shinohara M, Araki E, Kanazawa T, Suyehiro K, Mochizuki M, Yamada T, Nakahigashi K, Kaiho Y, Fukao Y (2006) Deep-sea borehole seismological observatories in the western Pacific: temporal variation of seismic noise level and event detection. Ann Geophys 49(2/3):625-641

Shinohara M, Kanazawa T, Yamada T, Nakahigashi K, Sakai S, Hino R, Murai Y, Yamazaki A, Obana K, Ito Y, Iwakiri K, Miura R, Machida Y, Mochizuki K, Uehira K, Tahara M, Kuwano A, Amamiya S, Kodaira S, Takanami T, Kaneda Y, Iwasaki T (2008) Precise aftershock distribution of the 2007 Chuetsu-oki Earthquake obtained by using an ocean bottom seismometer network. Earth Planets Space 60:1121-1126

Urabe T, Tsukada S (1991) A workstation-assisted processing system for waveform data from microearthquake networks. Abstracts of Spring Meeting of Seismological Society of Japan 70 (in Japanese)

Wessel P, Smith WHF (1991) Free software helps map and display data, EOS. Trans Am Geophys Union 72:441

Wilcock WSD, Webb SC, Bjarnason IT (1999) The effect of local wind on seismic noise near $1 \mathrm{~Hz}$ at the MELT site and on Iceland. Bull Seismol Soc Am 89:1543-1557

Yamada T, Mochizuki K, Shinohara M, Kanazawa T, Kuwano A, Nakahigashi K, Hino R, Uehira K, Yagi T, Takeda N, Hashimoto S (2008) Aftershock observation of the Noto Hanto earthquake in 2007 using ocean bottom seismometers. Earth Planets Space 60:1005-1010

Yamazaki K, Yamamoto H, Shinohara M, Kanazawa T (2012) Development of seismometers sensor network for observation on sea floor-IP goes to oceans-IEICE. Trans Commun E95-B 7:2182-2190 